Original Research Paper

\title{
Adsorption Behavior of Bone Morphogenetic Protein-2 on a Graphite Substrate for Biomedical Applications
}

\author{
Izabele Marquetti and Salil Desai \\ Department of Industrial and Systems Engineering, \\ North Carolina A\&T State University, Greensboro, USA
}

Article history

Received: 08-5-2018

Revised: 30-5-2018

Accepted: 13-6-2018

Corresponding Author:

Salil Desai

Department of Industrial and

Systems Engineering, North

Carolina A\&T State University,

Greensboro, USA

Email: sdesai@ncat.edu

\begin{abstract}
The interaction of proteins with biomaterials plays an important role for several biomedical applications including implants and tissue engineering. Although different experimental approaches have been explored, the investigation of the molecular models for protein-substrate interaction is warranted to control of cellular functions. In this study, Molecular Dynamics (MD) simulations were used to study the adsorption behavior of Bone Morphogenetic Protein-2 (BMP-2) on a hydrophobic graphite substrate. The influence of four different orientations of the protein with the substrate was evaluated based on their adsorption behavior. Results indicate a strong influence of the initial configuration on the adsorption. Protein unfolding was observed for most orientations with preferential binding of residues within the vicinity of the substrate. Most of the secondary structure of the protein was preserved during the simulation with a minor reduction of $\beta$-sheet structures. This research provides a detailed understanding of the interaction of BMP-2 with biomaterial surfaces.
\end{abstract}

Keywords: Biomaterials, Biomedical Implants, Bone Morphogenetic Protein-2, Graphite, Molecular Dynamics, Protein Adsorption

\section{Introduction}

Biomaterials have emerged as an alternative method to improve the performance of medical devices (Perkins et al., 2014; Gad and Gad-Mcdonald, 2015; Perkins et al., 2015). Implantable biomaterials demand certain characteristics such as adequate mechanical properties for bone replacement, the absence of foreign body interactions that adversely affect the tissue and in vivo biodegradability (Starý et al., 2003).

Protein adsorption onto a biomaterial and their interactions with tissue cells are essential to the biocompatibility of implants (Mücksch and Urbassek, 2011b). The surface properties of the biomaterial regulate the final confirmation of the protein concerning its bioavailability and cell signaling activities. Important functionalities can be obtained if the protein remains bioactive after adsorption (Utesch et al., 2011). The adsorption behavior of a protein depends on the interaction between the amino acids in a protein, the properties of the material surface and the conditions of the solution in which the material is solvated (Latour, 2009).
Bone Morphogenetic Protein-2 (BMP-2) is an important protein involved in different cellular functions, such as differentiation and proliferation of osteoblasts, promoting bone formation and regeneration (Oliveira et al., 2011a; Beederman et al., 2013). The use of BMP-2 combined with standard bone repair procedures can prevent rejection and reduce the healing time. However, to obtain the desired repair of the tissues, the protein must be coated on material without any conformational changes that affect its bioactivity (Oliveira et al., $2011 \mathrm{~b}$ ). For the effective bone restoration, protein adsorption on the surface material must be stable and occur at the implant site. Also, the protein should be able to interact with its receptors, activating signaling cascades and thus inducing osteoblasts proliferation (Utesch et al., 2011). A too strong binding of the protein on the implant leads to partial denaturation and consequently, affecting its bioactivity.

The design of biomaterials to attain favorable surface interaction with growth factors and nutrients is crucial for tissue engineering applications. Molecular Dynamics 
(MD) simulations are an effective method to understand the adsorption behavior of a protein, allowing an atomiclevel characterization of the conformational changes in a protein structure (Klepeis et al., 2009; Latour, 2009).

In this study, MD simulations were implemented to investigate the interaction behavior of BMP-2 with a hydrophobic graphite substrate. Pyrolytic graphite is a common material used in implants because of its high biocompatibility, low friction coefficient and adequate stiffness with bone (Mücksch and Urbassek, 2011a). However, its interaction with osteogenic growth factors lacks understanding of the molecular mechanisms that promote stem cell differentiation. In this research, the effect of four orthogonal orientations on the adsorption behavior of BMP-2 protein with graphite revealed preservation of secondary structures. This research plays a critical role in incorporating BMP-2 in biomedical implants for stem cell-based regeneration in orthopedic applications.

\section{Methods}

MD simulations were carried out using Nanoscale Molecular Dynamics (NAMD) (Phillips et al., 2005) with the CHARMM27 force field (MacKerell Jr et al., 1998). Visual Molecular Dynamics (VMD) was used to build the models and analyze the results (Phillips et al., 2005). The simulations were performed on a 64-bit Linux platform (Fedora 21) with two Graphical Processing Units (GPUs) from NVIDIA ${ }^{\circledR}$ Corporation (K40 and K20).

The protein crystallographic structure of the BMP-2 with 114 amino acids was obtained from the RCSB Protein Data Bank (Berman et al., 2000). This structure was solvated in a water sphere using explicit TIP3P model (Marquetti and Desai, 2016). Sodium chloride $(\mathrm{NaCl})$ ions were added at a concentration of $0.15 \mathrm{~mol} / \mathrm{L}$ using VMD plugin. This solvated model consisted of a
$6.5 \mathrm{~nm}$ drop with 13,863 atoms. The solvated protein was placed in four different orientations on the center of a flat hydrophobic graphite, large enough to allow the movement of the protein (Fig. 1).

The non-bonded force field parameters for graphite were defined as $\sigma_{i j}=3.195 \AA$ and $\epsilon_{i j}=-0.439 \mathrm{~kJ} / \mathrm{mol}$ (Werder et al., 2003) to reproduce the contact angle of $86^{\circ}$, while the parameters for the water molecules and the protein were obtained from the CHARMM force fields (MacKerell Jr et al., 1998). All graphite atoms were kept fixed for computational efficiency. In this CPU+GPU configuration, the CPUs compute the energy evaluations for MD simulations and the GPUs compute the non-bonded force evaluation (Kindratenko et al., 2009; Cordeiro and Desai, 2016).

The MD models were minimized for $0.2 \mathrm{~ns}$ and then executed for $20 \mathrm{~ns}$ using an integration time step of $2 \mathrm{fs}$. Data were recorded every $0.1 \mathrm{~ns}$. The cutoff distance for Van der Waals interactions was $12 \AA$. Periodic boundary condition was applied for $\mathrm{x}$ - and $\mathrm{y}$ - directions. A constant temperature of $310 \mathrm{~K}$ was ensured using the Langevin temperature control (Marquetti and Desai, 2018).

The time evolution of the root-mean-square deviation (RMSD) was calculated to determine if the system reached equilibration phase. In this phase, RMSD reaches a steady state value due to the absence of variation in the potential energy (Equation 1):

$$
R M S D=\sqrt{\frac{\sum_{i=1}^{N_{\alpha}}\left(r_{i}\left(t_{1}\right)-r_{i}\left(t_{2}\right)\right)}{N_{\alpha}}}
$$

Where:

$N_{\alpha}=$ The number of atoms whose position are being compared

$r_{i}(t)=$ The position of atom $i$ at time $(t)$

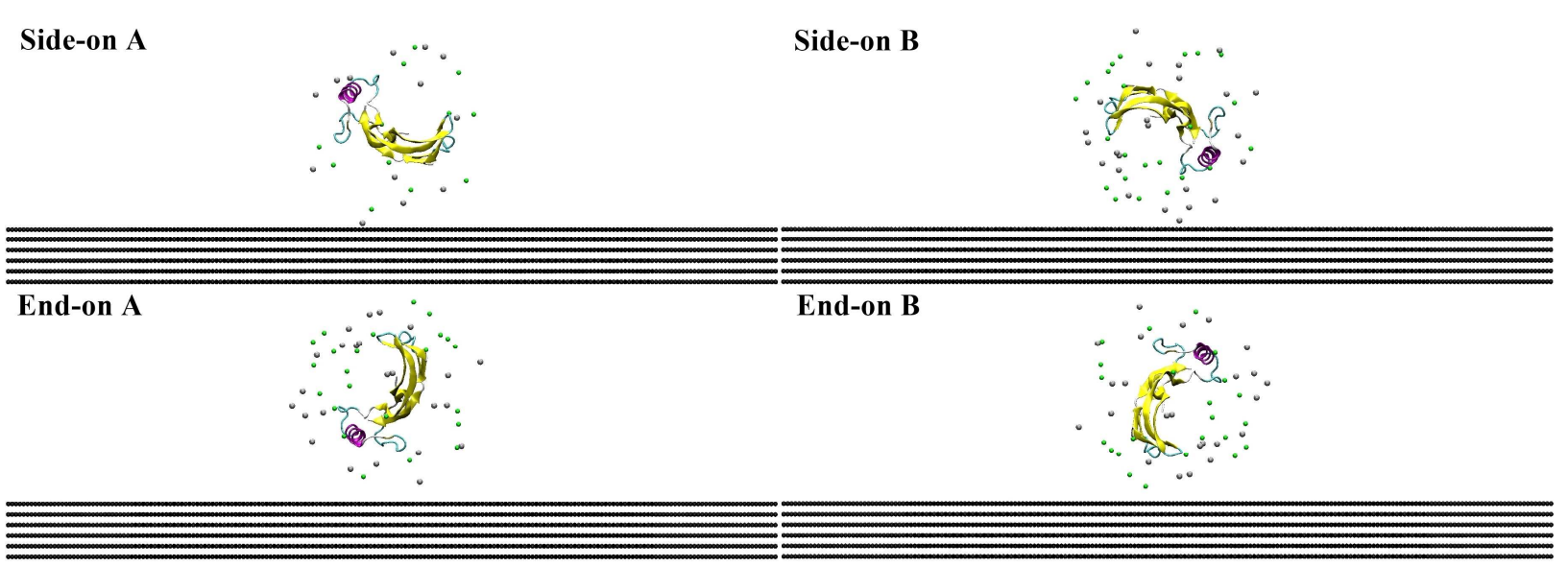

Fig. 1: Initial orientations of the BMP-2 placed on the graphite substrate (water molecules were excluded from the representation to better visualize the model). Protein is represented using secondary structure, $\alpha$-helix is represented in pink and $\beta$-sheets in yellow 
The radius of gyration of the BMP-2 was used to analyze the folding behavior of the protein structure during the simulations (Equation 2). This measure was used to evaluate the protein unfolding behavior. A significant folding and/or unfolding may lead to protein denaturation and loss of bioavailability of the BMP-2:

$$
R_{g}=\sqrt{\frac{\sum_{i}\left|r_{i}-r_{\text {com }}\right|^{2} m_{i}}{\sum_{i} m_{i}}}
$$

Where:

$\left|r_{i}-r_{\text {com }}\right|=$ The distance of the atom $i$ with mass
$m_{i}=$ The center of mass of the protein

\section{Results and Discussion}

Figure 2 presents the RMSD of the protein over time for all the protein orientations. The initial orientation influenced the RMSD values with mild fluctuations concerning time. The end-on A orientation had a rapid increase in the RMSD value within the initial $0.5 \mathrm{~ns}$ indicating protein unfolding behavior. This behavior was confirmed with an increase in the initial adsorption energy with the substrate. Similarly, the side-on B orientation had a slower increase in RMSD which corresponds to a delayed substrate adsorption with lower adsorption energy. Figure 3 shows no variation of the potential energy after the initial $0.5 \mathrm{~ns}$ confirming that the system reached equilibration for all the orientations.

Adsorption of BMP-2 on the substrate was measured by the non-bonded energy, which includes
Van der Waals and electrostatic energies of the interaction between protein and substrate (Utesch et al., 2011). Protein adsorption on graphite occurred exclusively via attractive Van der Waals interactions, which are weaker than electrostatic interactions, due to the lack of partial charges (Utesch et al., 2011). Initial adsorption occurred within a short time frame of $0.5 \mathrm{~ns}$ for all the protein orientations (Fig. 4). The orientation of the protein had an influence on its adsorption behavior with the hydrophobic graphite. End-on A configuration presented the strongest adsorption behavior with a rapid increase in the adsorption energy after $10 \mathrm{~ns}$ and reaching $-750 \mathrm{~kJ} / \mathrm{mol}$ at the end of the simulation. Side-on A and end-on B had the same adsorption behavior during the early stage of the simulation with a minor increase of the adsorption energy of the side-on A system $(-400 \mathrm{~kJ} / \mathrm{mol})$ after 9 ns. However, the side-on B orientation displayed weak adsorption until $10 \mathrm{~ns}$, followed by a rapid increase in the energy until it reached $(-325 \mathrm{~kJ} / \mathrm{mol})$ equivalent to the end-on B orientation.

The spreading of the water droplet on the hydrophobic substrate arranged the atoms of the protein with end-on orientations to a compact state (Fig. 5). End-on B orientation presented a folded structure, with the lowest values of radius of gyration (average 17.69 $\AA$ ). Side-on B spread out more on the surface (average $18.77 \AA$ A). Mücksch and Urbassek (2011a) have revealed that the side-on $\mathrm{B}$ orientation had more flexibility to spread out on the surface due to the weaker interaction energy at the beginning of the simulation. Our findings are consistent with results obtained by Mücksch and Urbassek (2011a) (Fig. 4).

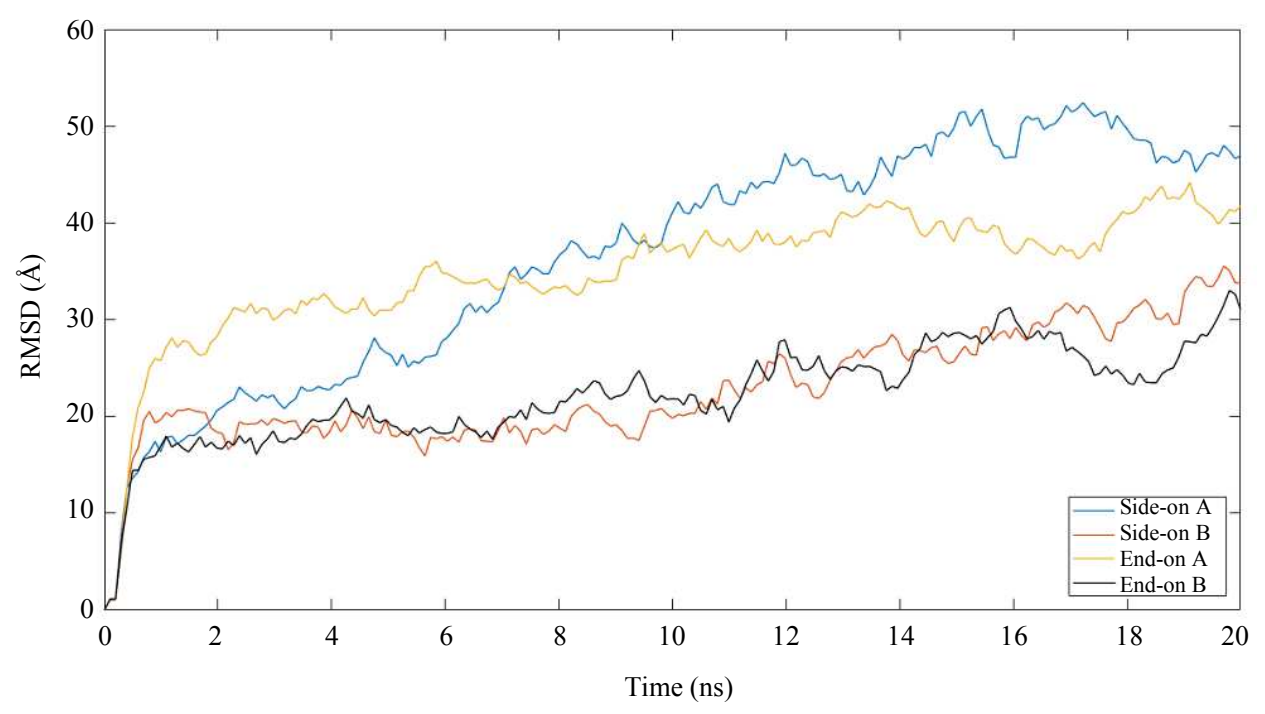

Fig. 2: Time evolution of the root-mean-square deviation 


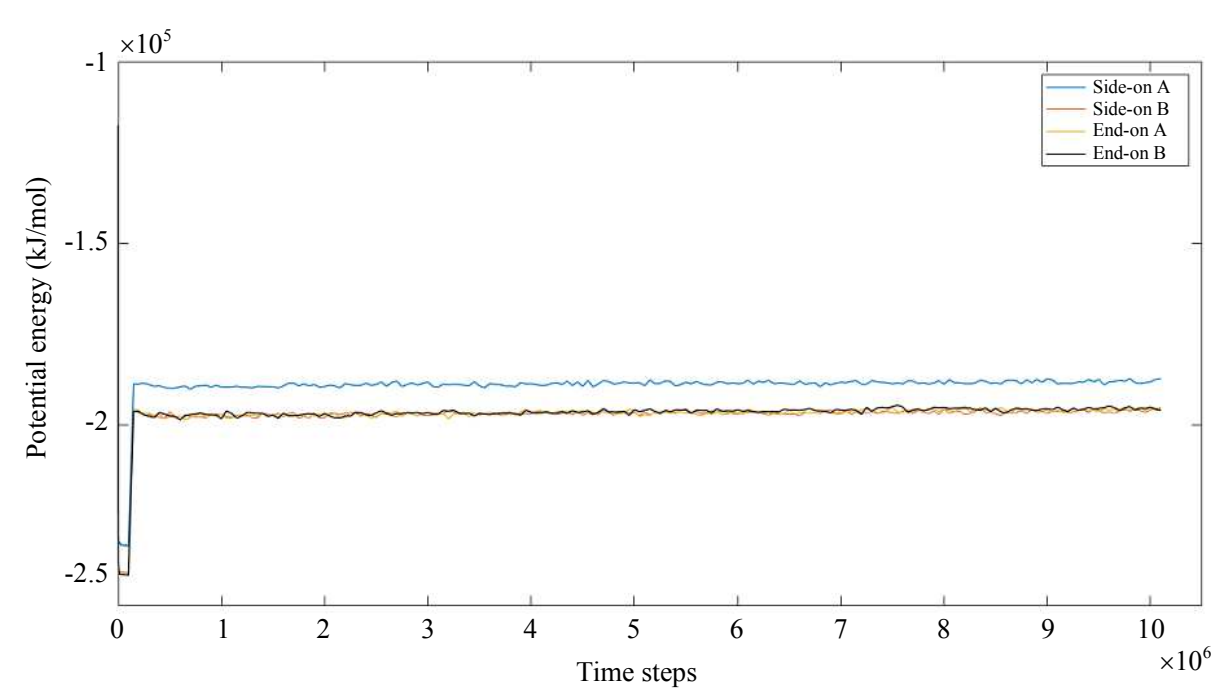

Fig. 3: Time evolution of the potential energy

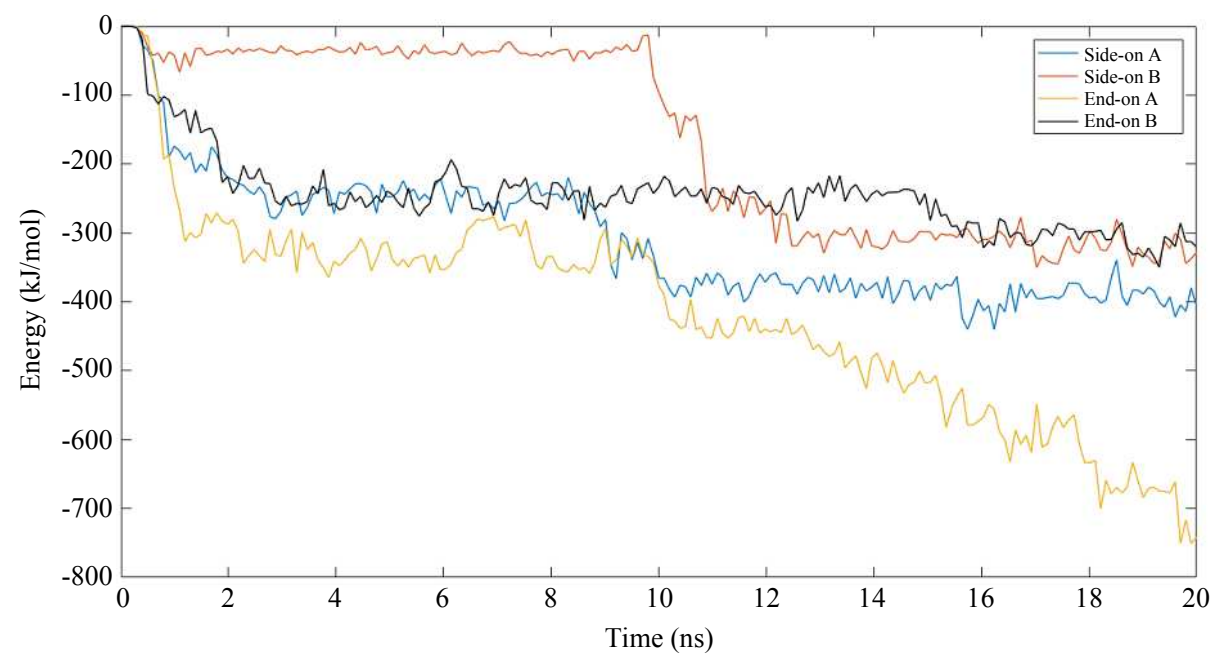

Fig. 4: Non-bonded energy between BMP-2 and graphite surface

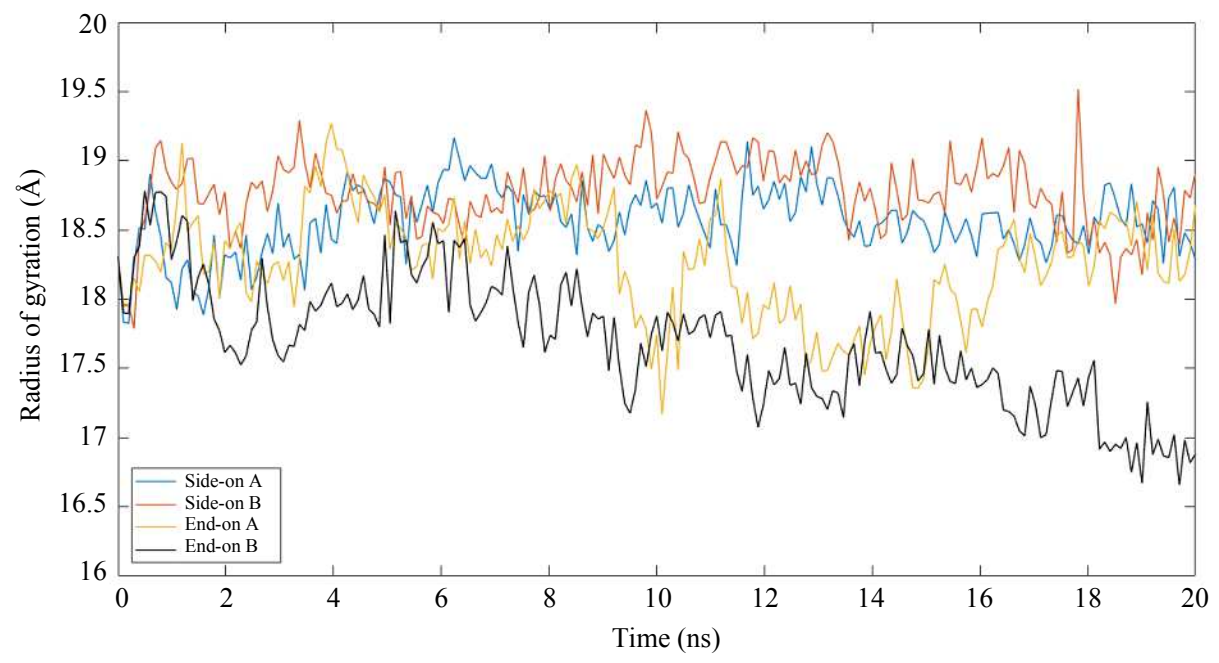

Fig. 5: Time evolution of the radius of gyration of the BMP-2 


\section{Side-on A}
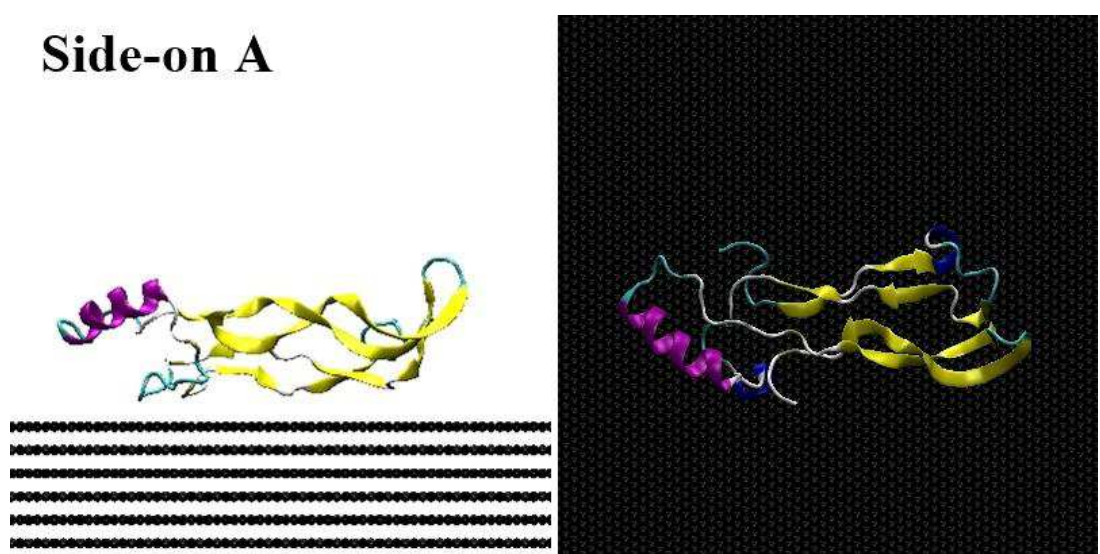

\section{Side-on B}
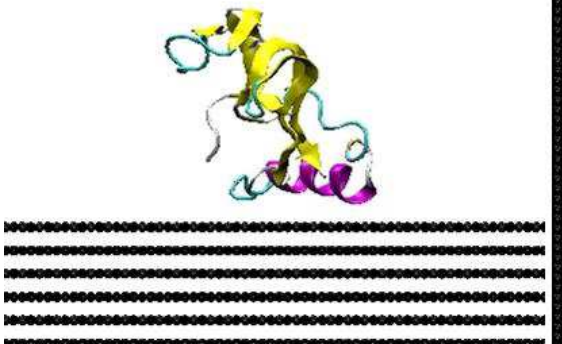

\section{End-on A}

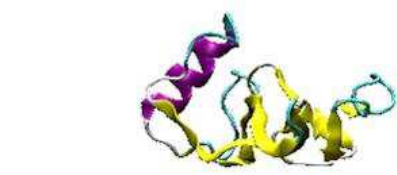

1.

-

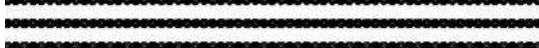

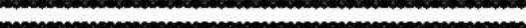

End-on B
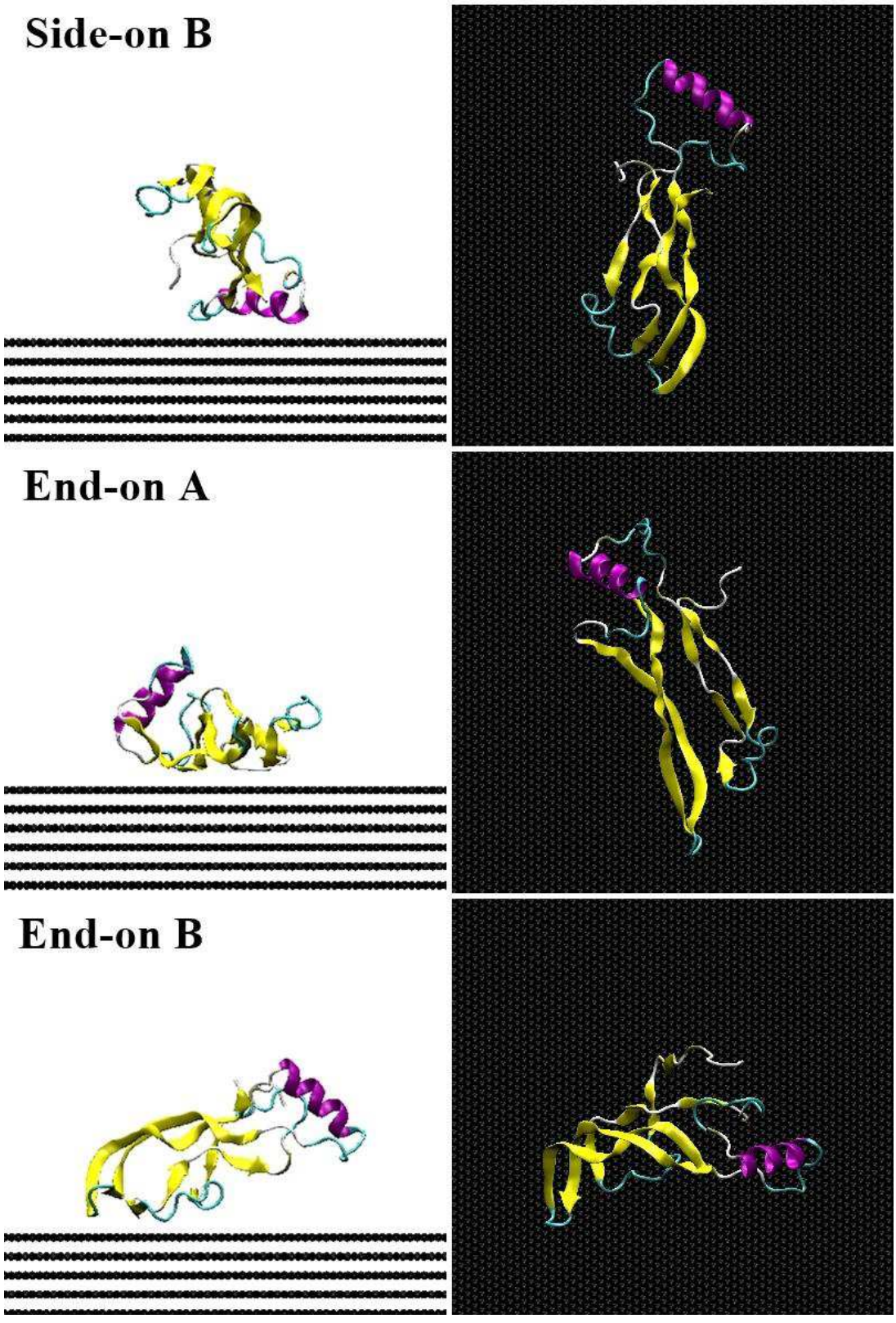

Fig. 6: Adsorption snapshots after 20 ns of simulation. Left: side view; right: Top view 
Table 1: The secondary structure of the BMP-2 at the beginning and the end of the simulations

\begin{tabular}{|c|c|c|c|c|c|}
\hline \multirow[b]{2}{*}{ Initial structure $(\%)$} & & \multicolumn{4}{|c|}{ After 20 ns (\%) } \\
\hline & & Side-on A & Side-on B & End-on A & End-on B \\
\hline$\beta$-sheet & 44.34 & 34.91 & 25.47 & 25.47 & 29.25 \\
\hline$\alpha$-helix & 10.38 & 11.32 & 15.09 & 11.32 & 11.32 \\
\hline 310-helix & 0.00 & 5.66 & 2.83 & 0.00 & 2.83 \\
\hline
\end{tabular}

Protein adsorption was measured by the number of contacts, which is the number of atoms in a residue that are within $5 \AA$ of the surface. Proteins constitute macromolecules formed by linking different types of amino acids. Residues are formed when two or more amino acids are combined and elements of water are removed from the peptide chain. Residues can be classified as polar, hydrophobic and charged (Schermann, 2008). Residues are typically represented by their threeletter abbreviations. Charged residues are more exposed to the solvent and can form salt bridges, influencing the stability of the protein (Sears, 2007). Polar residues tend to adsorb on hydrophilic surfaces while hydrophobic residues on hydrophobic surfaces (Dee et al., 2003; Schmidt et al., 2009). Therefore, these residue classes directly influence protein adsorption.

BMP-2 orientations have a strong influence on preferential adsorption of residues on the graphite substrate. Our findings show that residues which formed the lower part of each BMP-2 orientation tended to first approach the substrate and strongly adsorb in the initial stages of the simulations. The spreading of water molecules in the later stages subsequently enabled the adsorption of other prominent residues.

For side-on A orientation, the protein strongly adsorbed with more than 350 atoms in the polar residue TYR20. The other residues included charged ARG9 and hydrophobic PHE40 with more than 200 atoms. For endon A orientation, the polar residues HIS54 and ASN56 and charged ASP53 adsorbed first on the surface. This was followed by strong adsorption after $10 \mathrm{~ns}$ of charged residues ARG9, ARG114 and LYS11 with over 250 atoms per residue. In the end-on $\mathrm{B}$ orientation, the protein preserved its parallel orientation for a longer period. This permitted the adsorption of the residues within proximity of the graphite substrate and inhibited the contact of other residues. The main residues responsible for this adsorption were ASN29, ASP30 and hydrophobic residues VAL33 and PRO36, with 100 and 200 atoms, respectively. Side-on B orientation had only one residue (charged LYS73) adsorbing until $10 \mathrm{~ns}$. However, in the later stages of the simulation beyond 10 $\mathrm{ns}$, the BMP-2 rearranged from a parallel to a perpendicular orientation with the surface. Thereby, allowing polar HIS60, hydrophobic VAL63 and ILE74, with more than 150 atoms adsorbing per residue.

Protein denaturation was measured by the analysis of the secondary structure of the initial and final confirmation. Denaturation occurs when $\alpha$-helix and $\beta$-sheet structures are transformed into a random coil state (Smith et al., 1996). The changes in the secondary structures after $20 \mathrm{~ns}$ are presented in Table 1 for all the orientations. A reduction of the $\beta$-sheet content occurred for all BMP-2 configurations. The reduction was particularly higher for side-on $\mathrm{B}$ and end-on A orientations. A minor increase in $\alpha$-helix structures was observed in all simulations. Also, 310helix structures. 310-helix structures were formed in side-on A (5.66\%), side-on B (2.83\%) and end-on B $(2.83 \%)$ orientations, respectively.

Figure 6 presents the side and top view of the adsorption process after $20 \mathrm{~ns}$. For all the orientations except side-on B, the spreading of the water molecules prevented the $\alpha$-helix loop to contact the substrate, thereby preserving the $\alpha$-helix structures during adsorption. The final confirmation of the protein clearly depends on the initial orientation of the protein because the lower residues in close proximity with the substrate in each configuration adsorbed first. For the side-on B configuration, even though most of the $\alpha$-helix adsorbed on the surface, a significant reduction of the $\beta$-sheets occurred after 12 ns.

\section{Conclusion}

In this study, our group investigated the adsorption behavior of BMP-2 on hydrophobic graphite using MD simulations. The influence of four initial orthogonal orientations (two side-on and two end-on) on the protein adsorption was evaluated. The adsorption process on hydrophobic graphite occurred exclusively via Van der Waals interactions with the protein. Therefore, the sideon B orientation with lower initial adsorption energy had higher values of radius of gyration due to its flexibility to spread out on the surface. The end-on B presented a compact structure, limiting protein adsorption because most of its residues were inhibited from contact with the graphite substrate. Different BMP-2 residues were responsible for the interactions with the surface, which revealed a strong influence of the initial orientation on the adsorption behavior of the protein. A stronger binding of the polar, charged and hydrophobic residues occurred with the surface for side-on A and end-on A orientations. For all the orientations, the protein preserved most of its secondary structure. However, a reduction of the $\beta$-sheets was observed, especially for 
side-on $B$ and end-on A configurations. The preservation of the $\alpha$-helix structures related with the incapability of the residues in the loop to approach the surface, thereby avoiding adsorption. This research lays the foundation for identifying BMP-2 orientations which can preserve the bioavailability to aid effective proliferation and differentiation of stem cells for regenerative tissue engineering.

\section{Acknowledgment}

This work was funded by US National Science Foundation (NSF CMMI: Award 1663128). In addition, we would like to thank CNPq (National Council for Scientific and Technological Development-Brazil) for the "Science without Borders" fellowship.

\section{Author's Contributions}

Izabele Marquetti and Salil Desai: Performed simulation work and conducted an analysis of the research. All authors contributed to the writing of the manuscript.

\section{Ethics}

The authors declare that there are no ethical issues that could arise after the publication of this study.

\section{References}

Al-Karadaghi, S., 2017. The 20 amino acids and their role in protein structures. Structural Bioinformatics, Protein Crystallography, Sequence Analysis and Homolog Modeling.

Beederman, M., J.D. Lamplot, G. Nan, J. Wang and X. Liu et al., 2013. BMP signaling in mesenchymal stem cell differentiation and bone formation. J. Biomed. Sci. Eng., 6: 32-52. DOI: $10.4236 /$ jbise.2013.68A1004

Berman, H.M., J. Westbrook, Z. Feng, G. Gilliland and T.N. Bhat et al., 2000. The protein data bank. Nucleic Acids Res., 28: 235-242. www.rcsb.org

Cordeiro, J. and S. Desai, 2016. The leidenfrost effect at the nanoscale. J. Micro Nano-Manufactur., 4: 041001-041001. DOI: 10.1115/MSEC2016-8650

Dee, K.C., D.A. Puleo and R. Bizios, 2003. An Introduction to Tissue-Biomaterial Interactions. 1st Edn., John Wiley and Sons, Hoboken, ISBN-10: 0471461121, pp: 248.

Gad, S.C. and S. Gad-Mcdonald, 2015. Biomaterials, Medical Devices and Combination Products: Biocompatibility Testing and Safety Assessment. 1st Edn., CRC Press, Boca Raton, ISBN-10: 1482248387, pp: 584.
Kindratenko, V.V., J.J. Enos, G. Shi, M.T. Showerman and G.W. Arnold et al., 2009. GPU clusters for high-performance computing. Proceedings of the IEEE International Conference on Cluster Computing and Workshops, Aug. 31-Sept. 4, IEEE Xplore Press, New Orleans, LA, USA, pp: 1-8. DOI: 10.1109/CLUSTR.2009.5289128

Klepeis, J.L., K. Lindorff-Larsen, R.O. Dror and D.E. Shaw, 2009. Long-timescale molecular dynamics simulations of protein structure and function. Curr. Opin. Structural Biol., 19: 120-127. DOI: $10.1016 /$ j.sbi.2009.03.004

Latour, R.A., 2009. Molecular Simulation of ProteinSurface Interactions. In: Biological Interactions on Materials Surfaces: Understanding and Controlling Protein, Cell and Tissue Responses, Puleo, D.A. and R. Bizios (Eds.), Springer, New York, pp: 69-95.

Mackerell Jr, A.D., D. Bashford, M. Bellott, R.L. Dunbrack Jr and J.D. Evanseck et al., 1998. Allatom empirical potential for molecular modeling and dynamics studies of proteins. J. Phys. Chem. B, 102: 3586-3616. DOI: $10.1021 /$ jp973084f

Marquetti, I. and S. Desai, 2016. Molecular modeling of biochemical cues using high performance GPU. Proceedings of the International Interdisciplinary Conference on Engineering Science and Management Held, Dec. 17-18, Goa, India, pp: 255-262. $\mathrm{http} / / \mathrm{www}$.innovativeresearchpublication.com/docu ments/goa/paper\%2040.pdf

Marquetti, I. and S. Desai, 2018. Molecular modeling of bone morphogenetic protein for tissue engineering applications. Institute of Industrial and Systems Engineers Annual Conference, Florida.

Mücksch, C. and H.M. Urbassek, 2011a. Adsorption of BMP-2 on a hydrophobic graphite surface: A molecular dynamics study. Chem. Phys. Lett., 510: 252-256. DOI: 10.1016/j.cplett.2011.05.036

Mücksch, C. and H.M. Urbassek, 2011b. Molecular dynamics simulation of free and forced BSA adsorption on a hydrophobic graphite surface. Langmuir, 27: 12938-12943.

DOI: $10.1021 / \mathrm{la} 201972 \mathrm{f}$

Oliveira, A.F., S. Gemming and G. Seifert, 2011a. Conformational analysis of aqueous BMP-2 using atomistic molecular dynamics simulations. J. Phys. Chem. B, 115: 1122-1130. DOI: 10.1021/jp106092d

Oliveira, A.F., S. Gemming and G. Seifert, $2011 \mathrm{~b}$. Molecular dynamics simulations of BMP-2 adsorption on a hydrophobic surface. Materialwissenschaft Werkstofftechnik, 41: 1048-1053. DOI: $10.1002 /$ mawe. 201000707

Perkins, J., Z. Xu, C. Smith, A. Roy and P. Kumta et al., 2015. Direct writing of polymeric coatings on magnesium alloy for tracheal stent applications. Annals. Biomed. Eng., 43: 1158-1165. DOI: $10.1007 / \mathrm{s} 10439-014-1169-3$ 
Perkins, J., H. Yi, S.H. Ye, W. Wagner and S. Desai, 2014. Direct write manufacturing of controlled release coatings for drug eluting cardiovascular stents. J. Biomed. Res. Part A, 102: 4290-4300. DOI: $10.1002 / \mathrm{jbm} . \mathrm{a} .35105$

Phillips, J.C., R. Braun, W. Wang, J. Gumbart and E. Tajkhorshid et al., 2005. Scalable molecular dynamics with NAMD. J. Comput. Chem., 26: 1781-1802. DOI: 10.1002/jcc.20289

Schermann, J.P., 2008. Amino Acids, Peptides and Proteins. In: Spectroscopy and Modeling of Biomolecular Building Blocks, Schermann, J.P. (Ed.), Amsterdam, Elsevier, ISBN-10: 0444527087, pp: 251-296.

Schmidt, D.R., H. Waldeck and W.J. Kao, 2009. Protein Adsorption to Biomaterials. In: Biological Interactions on Materials Surfaces, Puleo D. and R. Bizios (Eds.), Springer, pp: 1-18.

Sears, D., 2007. Protein Structure Prediction: Concepts and Applications. 1st Edn., Wiley Online Library.
Smith, L.J., K.M. Fiebig, H. Schwalbe and C.M. Dobson, 1996. The concept of a random coil: Residual structure in peptides and denatured proteins. Fold. Design, 1: R95-R106. DOI: $10.1016 / \mathrm{S} 1359-0278(96) 00046-6$

Starý, V.R., L. Bačáková, J. HorníK and V. ChmelíK, 2003. Bio-compatibility of the surface layer of pyrolytic graphite. Thin Solid Films, 433: 191-198. DOI: 10.1016/S0040-6090(03)00309-2

Utesch, T., G. Daminelli and M.A. Mroginski, 2011. Molecular dynamics simulations of the adsorption of bone morphogenetic protein-2 on surfaces with medical relevance. Langmuir, 27: 13144-13153. DOI: $10.1021 / \mathrm{la2} 2489 \mathrm{w}$

Werder, T., J.H. Walther, R.L. Jaffe, T. Halicioglu and P. Koumoutsakos, 2003. On the water-carbon interaction for use in molecular dynamics simulations of graphite and carbon nanotubes. J. Phys. Chem. B, 107: 1345-1352.

DOI: $10.1021 /$ jp0268112 